\title{
Teenagers with Mental Disability Lack Reproductive Education and Knowledge; Still, Many Have Had Sex
}

Teenagers with mental disability report receiving less sex education in school than do their nondisabled peers, and their parents are more often reluctant to discuss reproduction and sexual health with them at home. Meanwhile, a considerable proportion of such teenagers-in particular, males-have had sexual intercourse, often without their parents' knowing. These findings come from an analysis of data from the National Longitudinal Study of Adolescent Health. ${ }^{1}$

Researchers analyzed data collected in 1994-1995 from a nationally representative sample of U.S. adolescents in grades 7-12 and, for a subsample, one of their parents. Adolescents were broadly categorized by mental ability on the basis of scores on a standardized picture vocabulary test, which are roughly comparable to IQ scores. The analyses included the 308 respondents the researchers classified as "mildly disabled" (those scoring 55-69) and the 114 they considered "more severely disabled" (those with a score of 54 or lower); some analyses involved comparison with respondents of "average intelligence" (those scoring 91-110). The mean age of the adolescent participants was $16-17$ years. A significantly larger proportion of teenagers considered mentally disabled than of those considered average were nonwhite and came from socioeconomically disadvantaged families.

The adolescents were asked whether they had learned about AIDS or pregnancy in school and whether they had ever had sexual intercourse. Respondents aged 15 or older also answered questions measuring their knowledge of birth control and sex. Parents were asked about the degree to which they had discussed certain sex-related topics with their teenage child and about their attitudes on sex and sex education for their child. Parents were also asked whether they thought their child had ever had sexual intercourse, and the researchers compared the answers to this question with those of the teenagers.

A smaller proportion of adolescents with mental disability than of mentally average teenagers reported having learned about re- production and sexual health at school. For example, $94 \%$ of males and $92 \%$ of females with average intelligence said they had been taught about AIDS in the classroom, compared with $78 \%$ of males and $85 \%$ of females with mild mental disability, and 65\% and 59\% with more severe disability. Similarly, exposure to classroom instruction on pregnancy was more prevalent among teenagers with average intelligence ( $85 \%$ of males and $90 \%$ of females) than among teenagers with mental disability (55-67\% of males and $49-82 \%$ of females).

Responses on the parent survey indicated that teenagers with mental disability also receive less at-home instruction than do teenagers with average intelligence. Reports of never discussing birth control were less common among parents with a son or daughter of average intelligence (16\% of each) than among parents whose child had mild mental disability $(26-44 \%)$ or more severe mental disability $(61-62 \%)$. In addition, the proportions never discussing sex, pregnancy or sexually transmitted diseases with their adolescent differed significantly between parents with an average child (6-12\%) and those whose child had mild (17-27\%) or more severe (44-55\%) mental disability.

The parent survey presented the following potential reasons why parents might choose not to discuss sex and birth control with their child: They did not know enough about these topics to be able to talk about them, they would find these topics difficult to explain, their child would be embarrassed by such talk, their child could get the information from another source and talking about sex would encourage sexual activity. Parents were asked to rate the extent to which they identified with each of these justifications on a scale of $1-5$, in which one means strong agreement and five denotes strong disagreement. Scores for parents of a child with average intelligence, which ranged from 3.6-4.4, were generally higher than those for parents of a child with mental disability3.4-4.0 for parents of a child with mild mental disability and 2.8-3.8 for parents of a child with more severe disability.
Using the same five-point scale, parents of a child with mental disability generally registered less-strong opposition than did other parents to their child's being sexually active. The level of disagreement with a statement indicating that parents disapprove of their child's having sex at the current time was higher among parents of a son with severe mental disability than among those whose son was of average intelligence (2.2 vs. 1.7); it also was higher among parents whose daughter had mild disability than among those with a daughter of average intelligence (2.1 vs. 1.6). Parents whose son had mild mental disability disagreed less strongly than parents of a son with average intelligence that they approved of their child's having sex with a "special friend" (3.4 vs. 4.1).

Overall, teenagers with mental disability demonstrated significantly poorer knowledge of fertility and birth control than teenagers with average intelligence. On an 11-point scale indicating level of knowledge (in which zero is the lowest possible score and 10 the highest), male respondents with average intelligence scored a mean of 6.0, whereas those with mild and more severe mental disability scored 2.8 and 1.8 , respectively. For female participants, the mean scores were 6.2, 3.2 and 1.4, respectively.

Using responses from the parent and adolescent surveys, the researchers estimated that by age $16,24 \%$ of the male teenagers with mental disability were sexually experienced-6\% whose parent knew this and $18 \%$ whose parent was unaware. Using multinomial logistic regression, the researchers calculated that among male teenagers with mental disability, the risk for being sexually experienced despite a parent's assumption to the contrary was more than twice that for male teenagers with average intelligence (relative risk, 2.4). Among female teenagers with disability, approximately $3 \%$ had had sex by age 16 , and their parent knew this; $6 \%$ had had sexual experience, but their parent did not know. Compared with females of average intelligence, females with mental disability were found to have a nonsignificantly higher relative risk for having sex without their parent's knowing (2.6). 
According to the authors, this study demonstrates that youth with mental disability receive inadequate sex education in school and at home. They note that it is therefore "not surprising that mentally disabled boys and girls have largely incorrect knowledge of birth control and human fertility." The authors comment that the considerable proportion of adolescents with mental disability who are sexually active without their parents' knowledge are "particularly vulnerable to sexual health risks, unwanted pregnancy, and sexual exploitation." They conclude that a better understanding is needed of "parents' attitudes and the problems they encounter at home in providing sex education to their disabled children."-C. Coren

\section{REFERENCE}

1. Cheng MM and Udry JR, How much do mentally disabled adolescents know about sex and birth control? Adolescent \& Family Health, 2003, 3(1):28-38.

\section{Mothers of Many Newborns With HIV Missed Chances To Obtain Preventive Care}

Between 1996 and 2000, more than half of HIV-infected infants in a large pediatric HIV surveillance project were born to women who missed opportunities to obtain perinatal HIV prevention care- $18 \%$ to women who received no prenatal care, $29 \%$ to women who had prenatal care but were not tested for HIV before delivery, and 9\% to women who had HIV diagnosed during pregnancy but did not receive antiretroviral treatment. ${ }^{1}$ The risk of motherto-child transmission of the virus was sharply reduced among women who received any preventive intervention during pregnancy; it was virtually eliminated among those who were treated with zidovudine in combination with other antiretroviral drugs.

The multisite project, funded by the Centers for Disease Control and Prevention, collects medical record data on children born to HIV-infected women. It is partly hospital-based and partly population-based, and the children on whom it gathers data are representative of all children born to U.S. women with HIV. Analysts used data on the 4,755 singleton births documented by the project during 1996-2000 to examine trends in use of perinatal HIV prevention methods, mother-to-child transmission rates and missed opportunities for perinatal preventive measures.
Six in 10 mothers included in the analyses were black, three in 10 were Hispanic and most of the rest were white. Seventy-nine percent had Medicaid or other public insurance coverage for their children, $7 \%$ had private insurance and $4 \%$ had no coverage; for $10 \%$, insurance coverage was unknown. About half of mothers supplied information on drug use; 23\% overall reported using illicit drugs.

The proportion of women known to have received prenatal care rose from $79 \%$ of those who gave birth in 1996 to $88 \%$ of those who delivered in 2000; data were not available for $10 \%$ of women. The prevalence of cesarean delivery, which is recommended for women with HIV infection, also grew over the study periodfrom $20 \%$ to $48 \%$.

Among women who received prenatal care, 90\% in 1996 and 94\% in 2000 had HIV diagnosed during pregnancy; $78 \%$ and $87 \%$, respectively, had antiretroviral drugs prescribed to prevent perinatal transmission of the virus. Whereas $71 \%$ of preventive drug regimens prescribed in 1996 relied on zidovudine alone, only 9\% took that approach in 2000; the proportion combining zidovudine with other antiretroviral drugs increased from $6 \%$ to $70 \%$. Seventy-nine percent of all infants born in 1996 received zidovudine treatment, compared with $92 \%$ in 2000 .

Nine percent of infants born during the study period were HIV-infected, and $77 \%$ were not; the infection status of $14 \%$ could not be determined. Among the infected infants whose records included information on the services their mothers received during pregnancy, 44\% were born to women who had prenatal care, an HIV diagnosis during pregnancy and treatment to prevent transmitting the virus to their newborn. For the other 56\%, the opportunity to take preventive measures was missed because the mother either received no prenatal care (18\%), received prenatal care but not an HIV test (29\%), or received care that included an HIV test but not antiretroviral therapy (9\%). By comparison, among uninfected infants, only $16 \%$ had any missed opportunity for preventive care.

A greater proportion of women who reported illicit drug use than of those reporting no such use received no prenatal care (17\% vs. 3\%). Results of logistic regression analysis confirmed this association: When project site, year, and mother's race or ethnicity and insurance status were controlled for, users of illicit drugs were significantly more likely than nonusers to have received no prenatal care (odds ratio, 8.0).
In a second logistic regression analysis, adding prenatal interventions and type of delivery to the set of controls, the researchers examined the factors associated with motherto-child HIV transmission. The strongest associations were with prenatal interventions: Compared with the risk of transmission in the absence of HIV testing and antiretroviral therapy, the risk was significantly reduced if the woman had been tested and had used zidovudine alone during pregnancy (odds ratio, 0.1 ) and, especially, if she had been tested and taken a prenatal drug regimen combining zidovudine and other antiretroviral agents (0.08). The transmission risk was lower in each of the last three years of the study period than in the first two (0.5-0.6) but was not associated with any of the other factors examined.

While the analysts stress that perinatal HIV transmission is largely preventable through the use of recommended prenatal interventions, they also emphasize that failures of these interventions are not uncommon and that investigation into the causes of those failures is imperative. Given their findings on missed opportunities for and failures of preventive measures, they conclude that the "most important" challenge in preventing mother-to-child HIV transmission is to ensure that all women are offered HIV screening prenatally or, if their HIV status is unknown, during labor and delivery, and that "comprehensive interventions are then aggressively offered."-D. Hollander

\section{REFERENCE}

1. Peters V et al., Missed opportunities for perinatal HIV prevention among HIV-exposed infants born 1996-2000, Pediatric Spectrum of HIV Disease cohort, Pediatrics, 2003, 111(5):1186-1191.

\section{Providers Do Not Fully Use Adolescent Well-Care Visits To Discuss Sexual Health}

Although 58-60\% of U.S. high school students make preventive health care visits, far lower proportions of those doing so-43\% of females and $26 \%$ of males-discuss pregnancy or sexually transmitted disease (STD) prevention at one. According to an analysis using 1999 survey data from the Youth Risk Behavior Surveillance System, ${ }^{l}$ primary care providers are thus missing opportunities to counsel their adolescent patients. Among sexually experienced females, the odds of having such a discussion are significantly elevated among users of a hormon- 
al method (odds ratio, 4.4), those aged 18 or older (3.5), those who have had at least four sex partners (2.3) and blacks (2.0). Among sexually experienced adolescent males, however, no characteristic or sexual risk behavior is independently associated with the odds of having discussed STD or pregnancy prevention.

Using data from the nationally representative survey of more than 15,000 high school students in grades 9-12, the researchers performed a series of logistic regression analyses to assess the association between characteristics and behaviors and two outcomes-having visited a primary care provider for preventive health care in the past year and having discussed ways to prevent pregnancy or STDs, including HIV, at that visit.

Roughly half of the sample ( $48 \%$ of females and $52 \%$ of males) had had sexual intercourse by the time of the survey; males were slightly older than females the first time they had sex ( 14.6 years vs. 13.9 years). Among sexually experienced students, a higher proportion of males than of females had had at least four sex partners (37\% vs. $27 \%$ ) and had used a condom at last intercourse ( $66 \%$ vs. $54 \%$ ). Use of a hormonal method (i.e., the pill or injectable) at last sex was more prevalent among females than among males ( $22 \%$ vs. $11 \%$ ).

Overall, 58-60\% of the students had visited a primary care provider for preventive health care in the past year (i.e., for a physical examination in the absence of sickness or injury). Among those who had made such a visit, 43\% of females and $26 \%$ of males said they had discussed pregnancy or STD prevention with their provider. Among sexually experienced students, $64 \%$ of females and $54 \%$ of males had gone for preventive care; $61 \%$ of females receiving such care discussed pregnancy and STD prevention at their visit, as did 34\% of similar males.

According to the logistic regressions, residence in the Northeast was independently and positively associated with having made a preventive health care visit among all females (adjusted odds ratio, 1.7), as was having initiated sexual activity (1.3); being Hispanic was associated with significantly reduced odds of having gone for preventive care (0.7). The odds of having discussed pregnancy and STD prevention at that visit were significantly elevated among females who had had intercourse (3.8), blacks (2.2) and older adolescents (odds ratios of 4.3 for those aged 18 or older and 2.9 for 17-year-olds).

Among sexually experienced females, residence in the Northeast was associated with in- creased odds of having made a preventive health visit (1.9), as was having had 2-3 sex partners (1.6) and having used a hormonal contraceptive at last sex (2.6). The odds of having discussed pregnancy and STD prevention at a recent visit were significantly elevated among sexually experienced females who had used a hormonal method at last coitus (4.4), who were aged 18 or older (3.5), who had had at least four partners (2.3) and who were black (2.0).

For males, being Hispanic and having initiated sexual activity were significantly associated with reduced odds of having seen a primary care provider for preventive care ( 0.6 and 0.8 , respectively). The odds of having discussed ways to prevent pregnancy and STDs with a provider were significantly elevated among males who had initiated sexual activity (1.9).

Among sexually experienced males, those living in the Northeast had significantly increased odds of having received preventive care (1.7), as did those whose partner used a hormonal method (1.5). No characteristic or behavior of sexually active adolescent males had an independent association with the odds of discussing pregnancy or STD prevention with a provider.

The researchers note that although half of U.S. high school students are sexually active and even higher proportions have made a recent preventive health visit, primary care providers do not use these opportunities to routinely discuss sexual and reproductive health. The students most likely to have discussed sexual and reproductive health were older, sexually experienced females who had already entered the health system and were using a hormonal method. Efforts to better use these clinical opportunities should focus on adolescent male patients, because condom use does not draw sexually active males into the health care system. The researchers acknowledge that their study is limited by the data's cross-sectional nature, the sampling of in-school youth only and the lack of information on other potentially important factors. Because the consequences of risky sexual behaviors are preventable, the authors conclude by recommending that "all primary care providers discuss HIV infection, STDs and pregnancy with their adolescent patients." -L. Remez

\section{REFERENCE}

1. Burstein GR et al., Missed opportunities for sexually transmitted diseases, human immunodeficiency virus, and pregnancy prevention services during adolescent health supervision visits, Pediatrics, 2003, 111(5):9961001.

\section{Maternal Mortality Exceeds U.S. Goal; Age and Racial Differences Are Marked}

During the period 1991-1999, more than 4,000 U.S. women died from pregnancy-related causes; in all, 11.8 maternal deaths per 100,000 live births were pregnancy-related. ${ }^{1}$ Analyses conducted by the Centers for Disease Control and Prevention (CDC) reveal that over the nine-year period, the pregnancy-related mortality ratio rose; the risk of pregnancy-related death was sharply elevated among women aged 35 or older and among black women. Maternal mortality remains substantially higher than the government's target for the year 2010.

Analysts from the CDC used data from the Pregnancy Mortality Surveillance System, which includes information on all pregnancy-related deaths reported by state health departments, maternal mortality review committees, media and individual providers. Birth certificates or fetal death certificates yielded additional data for the majority of women who had had a live birth or a stillbirth. The analysts classified a death as pregnancy-related if it occurred during or within one year after a pregnancy and resulted from complications of the pregnancy, events triggered by the pregnancy or the pregnancy's aggravation of an unrelated condition.

A total of 4,200 maternal deaths during the surveillance period were pregnancy-related. The pregnancy-related mortality ratio for the entire period was 11.8 deaths per 100,000 live births; the ratio rose from 10.3 to 13.2 per 100,000 between 1991 and 1999, and the increase was statistically significant. Women younger than 30 had below-average pregnancy-related mortality ratios (8.6-9.6 per 100,000 ), but the ratio was just above average for those in their early 30s and rose dramatically thereafter (to 21.6 for women in their late 30 s and 45.4 for those aged 40 and older). The analysts calculated risk ratios, which confirmed that women aged 30-34 had a modestly higher risk of dying from a pregnancy-related cause than women younger than 20 (1.4), and the risk was markedly elevated among women aged 35-39 (2.5) or older (5.3). Whereas white women had a pregnancy-related mortality ratio of 8.1 deaths per 100,000 live births, the ratio for black women was 30.0 per 100,000; the risk ratio (3.7) suggested that black women were almost four times as likely as white women to die from a pregnancy-related cause. 
Essentially the same racial differential in pregnancy-related mortality was evident across women's characteristics. Black women had a higher pregnancy-related mortality ratio than white women in every age-group studied. The gap widened as women's age increased, and in the oldest age-group (40 years or older), black women's ratio (nearly 160 per 100,000) was more than five times white women's (roughly 30 per 100,000). At all levels of education, the pregnancy-related mortality ratio among black women was 3-4 times that among their white counterparts. The same was true regarding the trimester in which women initiated prenatal care (although the differential was somewhat reduced among women who had received no care at all), and the pattern was similar regardless of the number of births a woman had had. Women in general and white women were less likely to die as a result of pregnancy if they were married than if they were not, but the reverse was true for black women.

Sixty percent of women who died from a pregnancy-related cause had had a live birth, and 10\% had not yet delivered; $4-7 \%$ had had a stillbirth, an ectopic pregnancy or an abortion, and the outcomes for 13\% were unknown. A higher proportion of black women than of white women had had an ectopic pregnancy ( $8 \%$ vs. $4 \%$ ); other pregnancy outcomes were comparable in the two racial groups.

The most common causes of pregnancyrelated maternal mortality were embolism (accounting for $20 \%$ of deaths), hemorrhage (17\%) and pregnancy-induced hypertension (16\%), but substantial variation was evident by pregnancy outcome. For example, the leading cause of death was embolism among women who had had a live birth (21\%), hemorrhage among those who had had a stillbirth (21\%) and infection among those who had had a spontaneous or induced abortion (34\%). Nearly all deaths related to an ectopic pregnancy (93\%) had resulted from hemorrhage; women who died while pregnant most often had had medical problems such as cardiovascular, pulmonary or neurologic conditions (34\%).

The analysts attribute the increase in pregnancy-related mortality in the 1990s to enhanced surveillance; nevertheless they note that while maternal deaths are rare, they remain underreported. As a result, they conclude that "substantial improvement" is needed to meet the government's Healthy People 2010 objective of reducing the level of maternal deaths to 3.3 per 100,000 live births-a goal that had also been set for the year 2000. They emphasize the need to further improve surveillance, identify the factors that contribute to excess maternal deaths among black women and "develop effective strategies to prevent pregnancy-related mortality for all women." $-D$. Hollander

\section{REFERENCE}

1. Chang J et al., Pregnancy-related mortality surveillance-United States, 1991-1999, Morbidity and Mortality Weekly Report, 2003, 52(SS-2).

\section{High Rates of Congenital Syphilis in Russia Linked To Inadequate Prenatal Care}

The incidence of congenital syphilis, which is largely preventable, is high in the Russian Federation. ${ }^{1}$ Because many infected pregnant women are treated inadequately or too late in their pregnancy, $64 \%$ deliver babies with presumptive or confirmed congenital syphilis. According to a review of records from five maternity hospitals in the Russian Federation, the odds of delivering a baby with congenital syphilis are elevated among infected women who go without prenatal care (odds ratio, 2.8) and among those who are first tested for syphilis at 28 weeks' gestation or later (4.0). Overall, $26 \%$ of the infants of women with untreated or inadequately treated syphilis die shortly after birth or in utero.

Women who were at least 20 weeks pregnant between January 1995 and October 1999 and delivered in maternity hospitals in two metropolitan areas (Moscow and St. Petersburg) and three nonmetropolitan areas (Novgorod, Moscow Oblast and Ryazan) were eligible for the study. (Deliveries included fetal deaths and induced abortions at 20 or more weeks' gestation.) A consecutive sampling of the hospitals' medical records yielded a sample of 850 women who had active syphilis, according to additional records from prenatal, women's health or sexually transmitted disease (STD) clinics. Active infections included new diagnoses during the index pregnancy and previous infections that had been untreated or inadequately treated (i.e., the woman was not given long- or short-acting penicillin within 30 days of delivery).

The researchers used results of clinical tests for confirmed congenital infection (physical examinations and serologic evaluations) and the World Health Organization definition of presumptive congenital infection, which includes any infant born to a woman with untreated or inadequately treated syphilis, to assess the rate of congenital infection according to maternal characteristics and pregnancy outcomes. They performed bivariate and multivariate logistic analyses to determine risk factors associated with congenital syphilis.

Most of the women (77-79\%) were married, were aged 20 or older, and resided in the cities where they sought care. Thirty-six percent each were homemakers and were unemployed; $12 \%$ described themselves as professionals, $10 \%$ as manual laborers and $5 \%$ as students. Fifty-seven percent of the sample had had inadequate prenatal care: Forty percent had had none at all, and $17 \%$ had initiated care at 21 weeks' gestation or later. A substantial proportion of the women-45\%-had first been tested for syphilis at 28 weeks' gestation or later; the same proportion had not received any antibiotic treatment for their infection. Further, $59 \%$ had not received the preferred antibiotic, penicillin G; the proportion not receiving it was $77 \%$ among women who had not received any prenatal care. Most of the infected women had an early latent infection (59\%), whereas 34\% were diagnosed with secondary syphilis and $6 \%$ with primary syphilis.

Overall, $64 \%$ of the infected pregnant women delivered an infant with presumptive or confirmed congenital syphilis. This proportion was significantly elevated among women who had not had any prenatal care (86\%), who were nonresidents (83\%) and who were first tested for syphilis at 28 weeks' gestation or later (83\%). There were significant trends both toward increasing incidence with later prenatal care $(41 \%$ among infants of women who sought care at 20 weeks or earlier vs. $63 \%$ among infants of women who obtained care at 21 weeks or later) and toward decreasing incidence with the number of prenatal visits (78\% among infants of women who made three or fewer visits vs. 53\% among infants of women who made at least four). Further, the incidence of congenital syphilis was significantly higher among babies of women with late latent syphilis than among those of women with primary or secondary syphilis ( $67 \%$ vs. $42-48 \%$ ). Finally, the rate of infection differed significantly by the setting in which the mother was diagnosed (48\% among infants of women diagnosed in an STD clinic, $60 \%$ among those of patients diagnosed in women's health clinics and $82 \%$ among those 
of women seen in other institutions).

Among all infected women for whom pregnancy outcomes were known, 65\% had a live birth, $15 \%$ an induced abortion, $11 \%$ a stillbirth, $6 \%$ a late fetal death (at 20 or more weeks' gestation) and $2 \%$ a liveborn baby who later died. Among women whose infant met the case definition for presumptive congenital syphilis, $26 \%$ had an outcome of fetal or infant death$16 \%$ had a stillbirth, $7 \%$ a baby who died in utero at 20 weeks' gestation or later, and 3\% a baby who was born alive and later died.

Results of the multivariate analysis, which controlled for the mother's age, residence and marital status, indicate that three factors independently increased the odds that an infected woman would deliver an infected infant. These were lack of prenatal care (adjusted odds ratio, 2.8), first being tested for syphilis late in the pregnancy (4.0) and having latent syphilis, as opposed to primary or secondary syphilis (3.7).
The investigators acknowledge that their study is limited by its retrospective design, the lack of data on substance abuse and a possible bias caused by the inclusion of women who were false positives (which would have underestimated the true incidence of congenital syphilis). They note that congenital syphilis "should be largely preventable" and that the "mainstay" of prevention-early diagnosis and appropriate treatment-needs to be strengthened among the range of Russian health care institutions that provide prenatal care. They conclude by noting that this recommended prevention strategy and infrastructure will also be essential for controlling perinatal transmission of HIV, which is expected to increase in the Russian Federation.-L. Remez

\section{REFERENCE}

1. Tikhonova L et al., Congenital syphilis in the Russian Federation: magnitude, determinants and consequences, Sexually Transmitted Infections, 2003, 79(2):106-110.

\section{Emergency-Room Visits Can Be Good Opportunities For Sexually Transmitted Disease Screening}

Screening emergency department patients for sexually transmitted diseases (STDs) can help identify undiagnosed infections, but because populations vary, screening programs have to be tailored to particular settings. ${ }^{1}$ At two Baltimore emergency departments, one in the inner city and one in another urban area, comparable proportions of patients who agreed to be screened tested positive for gonorrhea or chlamydia, but most of the risk factors associated with infection varied. In the inner-city facility, patients aged 25 or younger, individuals with a history of STDs and those who had recently had multiple sex partners had elevated risks of infection; in the other facility, women and those reporting multiple recent partners were at increased risk. Factors associated with declining to be screened also varied between populations.

The inner-city emergency department is in an area of Baltimore with a high STD incidence and provides care to a largely black, underserved population aged $18-44$. By contrast, the non-inner city facility is in a part of the city with a moderate incidence of STDs, and serves a clientele who are mainly white and aged 14-39. Men and women seeking medical treatment who were not critically ill and were able to give consent were eligible for study participation; the study was conducted in 1998 in the inner-city facility and in 2000 at the urban site. Participants completed interviews covering demographic and behavioral characteristics, and provided urine samples, which were tested for gonorrhea and chlamydia by ligase chain reaction.

In all, 454 patients aged $18-31$ visiting the inner-city facility (77\% of those approached during the study period) and 298 visiting the urban site $(61 \%)$ participated in the screening program. (The researchers focused on 18-31-year-olds because most infections occur in this group.) On average, individuals in both populations were about 24 years of age. The inner-city population had a higher proportion of women than the urban population (63\% vs. $52 \%$ ), as well as a higher proportion of black patients (90\% vs. 37\%). Nearly three in five participants in each setting had health insurance, but those in the inner city were more likely than others to say that they used the emergency department as their regular source of health care ( $58 \%$ vs. $48 \%$ ).

Fourteen percent of participants in each setting tested positive for gonorrhea or chlamydia, but several significant differences emerged between settings in behavioral factors for disease. Patients at the urban emergency department scored higher than inner-city men and women on a standard instrument assessing al- cohol use and were more likely to give positive responses to at least two questions on this instrument (17\% vs. $10 \%$ ); they were less likely to report having had a new sex partner in the past 90 days ( $16 \%$ vs. $26 \%$ ) and to say that they had ever had an STD (31\% vs. 52\%). Marginally higher proportions of participants at the inner-city site than at the urban facility were visiting the emergency department because of a genital discharge (6\% vs. $3 \%$ ) and received treatment for gonorrhea or chlamydia at their initial visit ( $10 \%$ vs. $6 \%$ ).

Results of multivariate logistic regression analyses revealed that the factors associated with 18-31-year-old patients' likelihood of testing positive for gonorrhea or chlamydia differed in the two settings. Among inner-city patients, the odds of testing positive were roughly doubled among men and women aged 25 or younger, those who had ever had an STD and those who had had two or more sex partners in the past 90 days (odds ratios, 2.0-2.2). Participants at the urban facility who reported multiple recent partners also were at increased risk of infection (odds ratio, 2.8); the only other significant predictor of infection in this population was being female (2.5).

Logistic regression analysis also demonstrated that factors associated with refusal to participate in STD screening at an emergency visit varied by setting. At the inner-city facility, the odds of refusal were significantly elevated among patients whose visit was not prompted by genitourinary symptoms (odds ratio, 2.8), and were reduced among black patients (0.5) and patients approached on particular days of the week (0.3-0.4). Patients at the urban site likewise had reduced odds of refusing if they were black (0.7) and increased odds of refusing if they were not seeking help for a genitourinary problem (1.7). In addition, women and participants aged 27-31 were more likely than men and younger patients to decline screening (1.6 and 1.5, respectively), and those approached during daytime hours were more likely to decline than those visiting the facility after six in the evening (2.2).

According to the investigators, given the prevalence not only of STDs but of substance use detected in this study, the emergency department "presents an intervention opportunity...for a number of public health and preventive services," especially for patients who use it as their regular source of health care. However, they caution that unmeasured or unmeasurable factors affect the results and generalizability of screening programs based in 
emergency departments. Therefore, they encourage an assessment of a given emergency department population "before implementation of a wide-scale screening program that uses parameters developed in another setting." -D. Hollander

\section{REFERENCE}

1. Mehta SD et al., Generalizability of STD screening in urban emergency departments: comparison of results from inner city and urban sites in Baltimore, Maryland, Sexually Transmitted Diseases, 2003, 30(2):143-148.

\section{Relative Risk of Cervical Cancer Rises with Duration Of Oral Contraceptive Use}

The relative risk of cervical cancer rises the longer a woman is on the pill. According to a meta-analysis based on data from 28 studies, ${ }^{1}$ women who use the pill for 10 or more years are 2.2 times as likely as never-users to develop cervical cancer, whereas the relative risks among users for 5-9 years and fewer than five years are 1.6 and 1.1, respectively. Among women infected with human papillomavirus (HPV), the virus associated with virtually all cases of cervical cancer, long-term pill users are 2.5 times as likely as never-users to develop cervical cancer. The results were broadly similar among the studies, despite the wide variation in the individual variables that were statistically controlled for

To assess the relationship between use of hormonal contraceptives (primarily the pill) and cervical cancer, the researchers pooled data from four cohort and 24 case-control studies published from 1986 through 2002 on users' risks of developing cervical cancer relative to that of never-users. The researchers examined relative risks of cervical cancer for three durations of pill use-fewer than five years (shortterm), 5-9 years (medium-term) and 10 or more years (long-term). Wherever possible, they categorized use as current or recently discontinued (i.e., within eight years) versus discontinued further in the past (i.e., eight or more years ago). Each of the 28 studies controlled for one or more of the following variables: the woman's HPV infection status, number of sexual partners, history of cervical screening, smoking and history of barrier method use. Some studies focused exclusively on the developed or the developing world, on HPVpositive or HPV-negative women, on invasive or in situ cervical cancers, or on squamous cer- vical cancers or adenocarcinomas of the cervix. Only the most fully adjusted data were used in the meta-analysis.

The studies, about half of which were conducted in the developing world, included 12,531 women with invasive or in situ cervical cancer. Among controls, the proportion reporting ever-use of the pill ranged from 19\% to $92 \%$ in the studies conducted in developed countries and from $25 \%$ to $65 \%$ in those carried out in developing countries; the proportion who took the pill for longer than five years was $18-62 \%$ in developed countries and $8-20 \%$ in developing countries.

For all 28 studies combined, the relative risk of cervical cancer rose with increasing duration of pill use: The risk of diagnosis among short-term pill users relative to never-users was 1.1, whereas the relative risk for medium-term users was 1.6, and that for long-term users was 2.2. The general finding of increasing risk with increasing duration of pill use was broadly similar among all 28 studies, irrespective of the control variables used.

Data from the 12 studies that assessed cervical cancer risk among HPV-positive women suggest that the relative risk rose with longer periods of use, but it was significantly elevated for long-term users only (2.5). The three studies that examined the association between injectable use and cervical cancer indicate that use of this method for five or more years was associated with a slight, marginally significant increase in cervical cancer risk (1.2).

Eleven studies had data on both how recently pill users had stopped their method and their total duration of use. These suggested that the risk of cervical cancer might decline after use is stopped. Among short-term users, women who were still using the pill or had stopped within the last eight years had a higher relative risk than those who had stopped at least eight years ago (1.4 vs. 1.1). Similarly, among long-term pill users, the relative risk of cervical cancer was 2.1 among women who were still on the pill or had gone off it more recently, whereas the relative risk among those who had stopped in the more distant past was 1.4 and only marginally significant.

According to the researchers, combining the studies' results indicated that cervical cancer risk rose with increasing duration of pill use "in virtually every way that the data were examined." They warn, however, that the variation in methodologies, study design and HPV measurement suggest that their summary indicators should be interpreted with caution.
Further, none of the studies controlled simultaneously for all potentially confounding variables, and although the data suggest that risk may decline after women stop hormonal contraceptive use, the researchers acknowledge that their meta-analysis was "hampered by the lack of published data cross-classifying women by duration of use and time since last use." Since the question of whether and how long effects of hormonal contraceptive use persist is "critical," to answer it they recommend that the data be reanalyzed with uniformly defined variables.-L. Remez

\section{REFERENCE}

1. Smith JS et al., Cervical cancer and use of hormonal contraceptives: a systematic review, Lancet, 2003, 361(9364):1159-1167

\section{Teenagers Have the Same Risks Whether Partners Are Sequential or Concurrent}

One-third of sexually active 15-18-year-olds have had more than one partner, either sequentially or concurrently, within the past 18 months, and these teenagers differ in important ways from their peers who have had only one partner during the same period. ${ }^{l}$ Furthermore, according to an analysis of data from the National Longitudinal Study of Adolescent Health, they are at greater risk for nonviral sexually transmitted diseases (STDs) than teenagers who have had a single recent partner: The odds of having an STD are more than doubled among those who have had sequential partners and nearly quadrupled among those who have had concurrent ones. These increases in risk, however, are statistically indistinguishable from each other.

The analysis included 4,707 participants in the first wave of the nationally representative survey who reported having had at least one heterosexual relationship in the previous 18 months. Using the reported dates of first and last intercourse with each partner during this interval, the analysts determined that $65 \%$ of these young people had had a single relationship, $21 \%$ sequential relationships and 14\% concurrent ones. All three groups were similar with respect to gender distribution and average age (17 years). The proportion of young people reporting concurrent relationships was significantly smaller among whites and Hispanics (13\% of each) than among blacks and 
members of other racial or ethnic groups (18\%).

On average, teenagers reporting sequential relationships had had 2.5 partners in the previous 18 months, and those reporting concurrent relationships had had 3.0. All pairwise differences in the number of partners among these adolescents and those who had had a single partner were statistically significant. Likewise, the three groups differed from each other in their levels of condom use at most recent intercourse $58 \%$ among those with only one partner, 55\% among those with sequential partners and $47 \%$ among those with concurrent partners) and in their confidence in their ability to use contraceptives (average scores, 10.8, 10.5 and 10.2, respectively, on a 15-point scale).

In other regards, the two groups reporting multiple partners differed from teenagers reporting one partner but not from each other: They were less likely to report use of a hormonal contraceptive at last intercourse (23-24\% vs. $28 \%$ ) and more likely to say they had regretted at least one sexual encounter because they had been drinking (27-31\% vs. 14\%).

Four percent of the sample reported having had a nonviral STD diagnosed in the past 12 months. The proportion was significantly lower among those who had had a single relationship (3\%) than among those who had had sequential or concurrent partners ( $5 \%$ and $7 \%$, respectively); the difference between the two groups with multiple partners was not statistically significant.

The analysts used logistic regression to identify factors that were independently associated with teenagers' nonviral STD risk. In a model that included relationship patterns but not number of partners, teenagers reporting sequential or concurrent relationships were at significantly greater risk for STDs than those reporting one relationship (odds ratios, 2.0 and 3.2, respectively); the risks for the two groups with multiple partners were not significantly different from each other. A second model, examining number of partners but not relationship patterns, showed that teenagers' STD risk increased by $36 \%$ for each additional partner they had had. Finally, when both relationship patterns and number of partners were included, the differential in STD risk between teenagers with one partner and those with sequential or concurrent partners increased slightly (odds ratios, 2.3 and 3.9, respectively), and the association with number of partners lost significance. Again, further analysis showed no difference in risk between those re- porting sequential and concurrent partners.

Results for background and other behavioral factors were similar in all three analyses. Women and blacks were at elevated risk for these infections (odds ratios, 3.1 and 3.5, respectively, in the most comprehensive model), as were adolescents who had used a hormonal contraceptive at last intercourse (1.7). In addition, as young people's confidence in their ability to use contraceptives increased, their STD risk rose (1.1).

Given the limitations of the study, including its cross-sectional design and the possibility that relationship types were misclassified, the analysts acknowledge that "to establish a causal linkage between sexual relationship patterns and STDs, a prospective study designed specifically to gather relationship information up to diagnosis of STD is needed." Nevertheless, they conclude that the results show the importance of interventions that "openly discuss the risks of concurrent sexual relationships" and the role of other behavioral and social risk factors. - D. Hollander

\section{REFERENCE}

1. Kelly SS et al., The role of sequential and concurrent sexual relationships in the risk of sexually transmitted diseases among adolescents, Journal of Adolescent Health, 2003, 32(4):296-305

\section{Method-Related Problems Account for Most Failures Of the Female Condom}

The female condom rarely breaks during use, but an efficacy study conducted in 1996-1998 indicates that slippage occurs in nearly one in 10 uses and women may be exposed to semen in up to one in five uses. ${ }^{1}$ Although a woman is most likely to be exposed if she and her partner experience mechanical problems with the condom, about half of exposures occur during uses in which couples do not have any problems. A separate set of analyses using the same data set shows that women also have an increased risk of being exposed to semen while using the female condom if they are in a shorterterm relationship, if there is a large disparity between the size of their vagina and the size of their partner's penis, and if intercourse is very active. $^{2}$

The study was conducted among women recruited from family planning clinics and a university population in Birmingham, Alabama. Women were eligible if they were aged 21-
49, were using an effective nonbarrier method of birth control, were in a mutually monogamous relationship, had intercourse at least six times per month, were at low risk for sexually transmitted diseases, had a uterus and had used tampons. Women were trained to use the female condom, collect samples of vaginal fluid with swabs before and after intercourse, fill out a form documenting any problems during condom use and record details of intercourse in a coital log. The women returned used condoms, which were inspected to confirm use and detect tears. Researchers assessed semen exposure by comparing concentrations of prostate-specific antigen (PSA) in vaginal fluid collected before and after intercourse.

A total of 210 women participated in the training. The median age in this group was 27 years, and the median monthly income per household member was $\$ 600$. Most of the women were white, were married and had completed college. Half had been in their current relationship for at least four years, and half had intercourse at least 12 times per month. None of the women had previously used the female condom.

\section{Method-Related Problems and Semen Exposure}

In analyses of 2,232 female condom uses by 175 participants who returned at least one condom, women reported either mechanical problems, which could lead to method failure, or acceptability problems, which could result in discontinuation, during $25 \%$ of uses. The most common mechanical problems were that the condom rode on the penis ( $7 \%$ of uses), the condom slipped out of the vagina (6\%) and the outer ring slipped inside the vagina (3\%). Condoms rarely broke (fewer than 1\% of uses). The most common acceptability problems were that the woman or her partner felt pain or discomfort ( $6 \%$ and $4 \%$ of uses, respectively) and the condom made noise (3\%). Overall, problems were more common during the first five uses than during subsequent uses; however, mechanical or acceptability problems still occurred in nearly one in 10 uses of the 20th condom.

The researchers studied semen exposure by examining data on 1,485 female condom uses that took place 24 hours or more after the previous act of intercourse, and for which PSA results were available; they used two criteria of exposure, to take into account random variations in PSA levels. According to these analyses, women were exposed to semen during $7-21 \%$ of uses, depending on the criterion used for exposure. In most cases, the quanti- 
ty of semen a woman was exposed to was minute. ${ }^{3}$ The rate of exposure was highest among women who experienced mechanical problems with the device (22-35\% of uses), markedly lower among those reporting acceptability problems (9-20\%) and lower still among those who had no problems (5-19\%). Nonetheless, at least $53 \%$ of exposures occurred during uses for which women did not report any problems.

Results of a multivariate analysis indicated that regardless of how exposure was defined, a woman had a significantly elevated risk of being exposed to semen if her partner's penis entered to the side of the condom, if semen leaked onto her, if the outer ring slipped into her vagina and if the condom broke. A woman's odds of exposure decreased slightly as she and her partner used more female condoms.

Noting that semen exposure, a novel measure of condom failure, has advantages over traditional measures, the researchers nevertheless point out that further studies are needed "to determine which semen exposure levels, as measured by PSA, predict the risk for pregnancy and [sexually transmitted disease] and to compare the female condom with the male condom."

\section{Partner Characteristics and Semen Exposure}

Characteristics of couples and intercourse associated with semen exposure were assessed in a multivariate analysis of 1,149 female condom uses by 100 women for which both coital log data and PSA data were available. These results showed that the odds of exposure to semen were about doubled if women reported that intercourse was more active than usual (odds ratio, 1.7) or if they had been in the relationship for less than two years (2.4). Women also had elevated odds of exposure (2.7) if they had a relatively large vagina (i.e., diaphragm size, 75-80 mm, as measured by a nurse practitioner at the beginning of the study) and their partner's penis was below the median size (as measured by the women, according to instructions they received at enrollment).

Further analyses revealed that women with a large vagina were at increased risk of having their partner's penis enter to the side of the condom (odds ratio, 3.0) and of having the condom's outer ring slip into their vagina (3.5). Women living in households in which the monthly income was more than $\$ 900$ per member were substantially less likely than women living in households with lower incomes to be exposed (0.3). In this analysis, a woman's odds of exposure to semen did not change as couples became more experienced using the female condom.

Commenting on the findings, the researchers note that even though the female condom may not offer perfect protection, it provides complete protection against semen in most uses. They add that couples with a large mismatch between vaginal size and penis size and couples having very active intercourse might want to consider methods of protection other than the female condom.-S. London

\section{REFERENCES}

1. Macaluso M et al., Efficacy of the female condom as a barrier to semen during intercourse, American Journal of Epidemiology, 2003, 157(4):289-297.

2. Lawson ML et al., Partner characteristics, intensity of the intercourse, and semen exposure during use of the female condom, American Journal of Epidemiology, 2003, 157(4):282-288.

3. Macaluso M et al., Macaluso et al. respond to "condom effectiveness and prostate-specific antigen," American Journal of Epidemiology, 2003, 157(4):301-302.

\section{Not All Ohio Physicians Offer HIV Testing During Standard Prenatal Care}

Fewer than half of physicians in northwest Ohio who treat women during pregnancy offer HIV testing to all their pregnant patients, according to a regional survey of obstetriciangynecologists and primary care physicians. ${ }^{1}$ Among those physicians who routinely offer HIV testing as part of standard prenatal testing, a greater proportion of obstetriciangynecologists than of family practitioners offer universal HIV testing (90\% vs. 53\%), whereas a greater proportion of internists (95\%) than of family practitioners (78\%) or obstetriciangynecologists ( $86 \%$ ) believe that HIV testing should be part of standard prenatal testing without pretest counseling or informed consent. In multivariate analysis, specializing in obstetrics and gynecology and having more than $60 \%$ of patients who accept HIV testing were significantly associated with physicians' offering HIV testing to all pregnant patients.

Using a database maintained by the Medical College of Ohio Physician Referral Service, researchers selected all physicians listed in northwest Ohio who specialized in obstetrics and gynecology, family medicine, internal medicine or other primary care fields. Between December 2000 and March 2001, selected physicians were sent a 20-question survey that asked about their demographic characteristics, practice demographics, and attitudes and practices regarding HIV testing in pregnancy. Physicians returned the surveys using a self-addressed, stamped envelope, and researchers analyzed the data using chi-square tests and multivariate logistic regression.

Overall, 1,116 surveys were mailed. Of the 261 physicians who returned an at least partially completed survey and provided care in any capacity to pregnant women, slightly more than half were aged $30-50,60 \%$ saw 50 or fewer pregnant women per year, and the same proportion had their practices located primarily in rural or suburban areas. Only 16\% reported predominantly seeing patients using Medicaid, and 5\% saw mainly Hispanic or black patients. Sixteen percent of physicians had seen at least one pregnant woman with HIV in their practice, and $8 \%$ had ever diagnosed HIV infection in a woman during prenatal testing.

Forty-two percent of physicians reported that they offer an HIV test as part of their standard prenatal testing; $16 \%$ of physicians offered HIV testing to a quarter or fewer of their pregnant patients. Reasons cited for not routinely testing for HIV were perceived low incidence of HIV in the practice (17\%), lack of patient acceptance (4\%), difficulty with paperwork or counseling for testing (3\% each), and lack of office staff or time (2\%). Among those physicians who routinely offered HIV testing as part of prenatal testing, a greater proportion of obstetriciangynecologists than of family practitioners said that they offer HIV testing to all of their pregnant patients (90\% vs. 53\%); a greater proportion of internists (95\%) than of family practitioners (78\%) or obstetrician-gynecologists ( $86 \%$ ) believed that HIV testing should be part of standard prenatal testing without pretest counseling or informed consent.

When asked about their attitudes toward HIV testing during pregnancy, 54\% of physicians believed that it should be a routine part of care, whereas 7\% (mainly family medicine physicians older than 40 practicing in rural areas) felt that it was not necessary for most women. One-third believed that including HIV testing in standard prenatal care would increase their ability to routinely test for HIV, and slightly more than one-third (37\%) felt that HIV testing in their practice would increase if they had brochures to aid pretest counseling. Forty-two percent of physicians agreed with the statement that women infected with HIV should not have children, whereas 3\% strongly disagreed. 
In chi-square analysis, several factors were significantly associated with physicians' offering HIV testing to all pregnant patients: specializing in obstetrics and gynecology; seeing 100 or more pregnant patients per year; being younger than 50 ; seeing primarily patients who use Medicaid; having more than $60 \%$ of patients accept HIV testing; having seen at least one pregnant patient with HIV; having made at least one HIV diagnosis through routine prenatal testing; and having a patient population that was at least $70 \%$ black. However, only specializing in obstetrics and gynecology and having more than $60 \%$ of patients accept HIV testing remained significant in multivariate analysis.

The researchers comment that prenatal HIV testing is the first step in preventing motherto-child transmission and provides an important "prevention education opportunity." However, as access to obstetrician-gynecologiststhe specialists who are the most likely to offer prenatal HIV testing to all their pregnant patients-may be limited in rural areas such as northwest Ohio, the researchers suggest that "nonobstetricians and primary care physicians also include universal HIV testing for pregnant women."-J. Rosenberg

\section{REFERENCE}

1. Duggan J et al., Survey of physician attitudes toward HIV testing in pregnant women in Ohio, AIDS Patient Care and STDs, 2003, 17(3):121-127.

\section{Become a Part of the Peer-Review Process}

All research published in Perspectives on Sexual and Reproductive Health is first reviewed by at least two experts in the field it represents. These peer reviewers are of immeasurable value to both the editors of the journal and the authors of the articles. If you are interested in joining our roster of peer reviewers, please e-mail your name, complete contact information, qualifications and areas of expertise (be as specific as you can) to kchiarello@guttmacher.org, or fax that information to Kaylynn Chiarello at 212-248-1951.

When we have a submission we would like you to review, we will contact you to discuss the paper's subject and a timetable for the review; we typically ask reviewers to send us their comments within two weeks. If you agree to review, we will send you, along with the paper, a form outlining the types of questions we would like you to consider as you read through the paper. Your expertise may extend to some but not all of these questions (for example, you may have solid "on-the-ground" experience in running a program but may not be qualified to comment on the statistical techniques used in evaluating one): That is fine, and it is part of the reason that we ask two reviewers to assess each paper.

We generally will not ask you to review for the journal more than once every six months, although if you express serious reservations about a paper and the author makes substantial changes in response to your comments, we may ask you to evaluate the revision. We acknowledge reviewers' contributions in a listing in the first issue of each volume. 